PROCEEDINGS OF THE

AMERICAN MATHEMATICAL SOCIETY

Volume 133, Number 5 , Pages 1423-1425

S 0002-9939(04)07771-8

Article electronically published on November 19, 2004

\title{
WEAKLY COMPACT OPERATORS INTO SEQUENCE SPACES: A COUNTEREXAMPLE
}

\author{
KARI YLINEN \\ (Communicated by N.Tomczak-Jaegermann)
}

\begin{abstract}
In a recent paper Gutiérrez and Villanueva have used, without giving a detailed proof, an analogue of a well-known result of Ryan characterizing the weakly compact operators from a Banach space $E$ into the space $c_{0}(X)$ of null sequences in a Banach space $X$. In this note a counterexample is given showing that in the statement of Gutiérrez and Villanueva an additional condition is needed.
\end{abstract}

\section{INTRODUCTION}

Let $E$ and $X$ be Banach spaces. Let $c(X)$ be the Banach space (with the supremum norm) of the convergent $X$-valued sequences and $c_{0}(X)$ its subspace consisting of the sequences converging to zero. For any Banach space $F$, we denote by $L(E, F)$ the space of the bounded linear maps $T: E \rightarrow F$. For $T \in L(E, F), T^{\prime}: F^{\prime} \rightarrow E^{\prime}$ is its adjoint and $T^{\prime \prime}$ its second adjoint.

If $n \in \mathbb{N}, \operatorname{pr}_{n}: c(X) \rightarrow X$ will denote the evaluation map at $n$, and we use the same notation for its restriction to $c_{0}(X)$. Any $\Phi \in L(E, c(X))$ determines the sequence of operators $\operatorname{pr}_{n} \circ \Phi \in L(E, X)$. Conversely, any sequence of operators

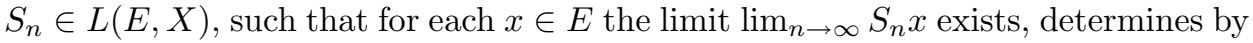
the uniform boundedness principle an operator $\Phi \in L(E, c(X))$ such that $\operatorname{pr}_{n} \circ \Phi=$ $S_{n}$. By a slight abuse of notation, we may write in short $\Phi=\left(S_{n}\right)$. In [4, p. 375] the following lemma is proved.

Lemma 1.1 (Ryan). A bounded linear map $\Phi: E \rightarrow c_{0}(X)$ is weakly compact if and only if the following two conditions hold:

(i) each operator $S_{n}=\operatorname{pr}_{n} \circ \Phi$ is weakly compact;

(ii) $\lim _{n \rightarrow \infty}\left\|S_{n}^{\prime \prime} z\right\|=0$ for each $z \in E^{\prime \prime}$.

Ryan's lemma has turned out to be very useful in the theory of multilinear operators, as is evident e.g. from the bibliography of [2]; see also [5]. Gutiérrez and Villanueva have published without a detailed proof an analogous statement (Lemma 3.3 in 2, p. 553]) in which $c_{0}(X)$ is replaced by $c(X)$ and condition (ii) by the requirement that the $\operatorname{limit}_{n \rightarrow \infty} \lim _{n}^{\prime \prime} z$ exists for each $z \in E^{\prime \prime}$. In [2], Lemma 3.3 is used repeatedly in crucial places, so it is unfortunate that this lemma is incorrect. We formulate as Proposition 2.1 a counterexample which shows this.

Received by the editors January 8, 2004.

2000 Mathematics Subject Classification. Primary 46B45.

Key words and phrases. Weakly compact operator, Banach sequence space, Yosida-Hewitt decomposition. 


\section{A Counterexample}

In the following proposition we denote $E=l^{1}$, the Banach space of functions $f: \mathbb{N} \rightarrow \mathbb{C}$ satisfying

$$
\|f\|_{1}=\sum_{n=1}^{\infty}|f(n)|<\infty .
$$

For $n \in \mathbb{N}$, let $S_{n}: E \rightarrow E$ be multiplication by the characteristic function of $\{1, \ldots, n\}$, i.e. $S_{n} f=\chi_{\{1, \ldots, n\}} f$. Clearly $\lim _{n \rightarrow \infty} S_{n} f=f$ for all $f \in E$. Let $\Phi: E \rightarrow c(E)$ be the bounded linear map satisfying $\operatorname{pr}_{n} \circ \Phi=S_{n}$ for all $n \in \mathbb{N}$.

Proposition 2.1. (a) Each $S_{n}: E \rightarrow E$ is weakly compact.

(b) For any $z \in E^{\prime \prime}$ the sequence $\left(S_{n}^{\prime \prime} z\right)$ is norm convergent.

(c) The operator $\Phi=\left(S_{n}\right)$ is not weakly compact.

Proof. Since the range of each $S_{n}$ is finite-dimensional, (a) is obvious.

To prove (b), choose $z \in E^{\prime \prime}$, i.e. $z$ is in the dual of $l^{\infty}$. Then $z$ may be identified with a finitely additive measure on the positive integers, see e.g. [1, p. 258], and we can write $z=\tilde{h}+\mu$ where $\tilde{h}$ is the canonical image of some $h \in l^{1}$ and $\langle\mu, g\rangle=0$ whenever $g \in l^{\infty}$ vanishes outside a finite set [3, p. 189]. (As noted in [3, p. 191], this is the Yosida-Hewitt decomposition of $\mu$.) Since

$$
\left\langle S_{n}^{\prime} g, f\right\rangle=\left\langle g, S_{n} f\right\rangle=\left\langle g, \chi_{\{1, \ldots, n\}} f\right\rangle=\sum_{k=1}^{n} g(k) f(k)=\left\langle\chi_{\{1, \ldots, n\}} g, f\right\rangle
$$

for all $f \in l^{1}, g \in l^{\infty}$, we have $S_{n}^{\prime} g=\chi_{\{1, \ldots, n\}} g$. Thus

$$
\left\langle S_{n}^{\prime \prime} z, g\right\rangle=\left\langle\tilde{h}+\mu, S_{n}^{\prime} g\right\rangle=\left\langle\tilde{h}, \chi_{\{1, \ldots, n\}} g\right\rangle+\left\langle\mu, \chi_{\{1, \ldots, n\}} g\right\rangle=\left\langle S_{n} h, g\right\rangle,
$$

i.e. $S_{n}^{\prime \prime} z=S_{n} h$, and so $\lim _{n \rightarrow \infty} S_{n}^{\prime \prime} z=h \in E \subset E^{\prime \prime}$ in norm.

To prove (c), simply observe that if $\Phi$ were weakly compact, then $\operatorname{pr}_{\infty} \circ \Phi$ would also be weakly compact, where $\operatorname{pr}_{\infty} \in L(c(E), E)$ is defined by $\operatorname{pr}_{\infty} f=$ $\lim _{n \rightarrow \infty} \operatorname{pr}_{n} f$. But this is impossible [1, p. 425], since $\operatorname{pr}_{\infty} \circ \Phi$ is the identity map on $E$, and $E$ is not reflexive.

To see Proposition 2.1 in perspective, we briefly consider a more general situation. Let $E$ again be a general Banach space, and $F$ a Banach space having $c_{0}(X)$ as a topological direct summand. Let $P_{1}: F \rightarrow F$ be a bounded projection onto $c_{0}(X)$ and write $P_{2}=I-P_{1}$ where $I$ is the identity map of $F$. The following observation is an immediate consequence of Lemma 1.1.

Proposition 2.2. A bounded linear map $\Phi: E \rightarrow F$ is weakly compact if and only if the following conditions hold:

(i) for each $n \in \mathbb{N}$, the operator $\operatorname{pr}_{n} \circ P_{1} \circ \Phi: E \rightarrow X$ is weakly compact;

(ii) for each $z \in E^{\prime \prime}, \lim _{n \rightarrow \infty}\left(\operatorname{pr}_{n} \circ P_{1} \circ \Phi\right)^{\prime \prime} z=0$;

(iii) the operator $P_{2} \circ \Phi$ is weakly compact.

Specializing to the situation considered by Gutiérrez and Villanueva, we choose $F=c(X)$. For $f \in c(X)$, let $P_{2} f$ be the constant sequence $k \mapsto \lim _{n \rightarrow \infty} f(n)$ and define $P_{1}=I-P_{2}$. Since both $P_{1}(F)=c_{0}(X)$ and $P_{2}(F)$ are closed, we have a topological direct sum here, i.e. $P_{1}$ and $P_{2}$ are bounded. In this case the canonical isometric isomorphism between $X$ and the space of the constant sequences in $X$ transfers $P_{2} \circ \Phi$ to the map $x \mapsto \lim _{n \rightarrow \infty} \operatorname{pr}_{n}(\Phi x)$. Thus Proposition 2.2 yields 
Corollary 2.3 below. We have seen that condition (iii) there cannot in general be dispensed with.

Corollary 2.3. A bounded linear map $\Phi: E \rightarrow c(X)$ is weakly compact if and only if the following three conditions hold:

(i) each operator $S_{n}=\operatorname{pr}_{n} \circ \Phi$ is weakly compact;

(ii) the limit $\lim _{n \rightarrow \infty} S_{n}^{\prime \prime} z$ exists for each $z \in E^{\prime \prime}$;

(iii) the operator $x \mapsto \lim _{n \rightarrow \infty} \operatorname{pr}_{n}(\Phi x)$ on $E$ is weakly compact.

Remark 2.4. It is a pleasure to acknowledge correspondence with J.D. Maitland Wright who pointed out that there was no obvious way of extending Ryan's result without assuming the above condition (iii). This served to motivate the work reported here.

\section{REFERENCES}

[1] N. Dunford and J.T. Schwartz, Linear Operators I, Interscience, New York (1958). MR0117523 $(22: 8302)$

[2] J.M. Gutiérrez and I. Villanueva, Extensions of multilinear operators and Banach space properties, Proc. Roy. Soc. Edinburgh 133A (2003) 549-566. MR.1983686 (2004c:46078)

[3] A. Olubummo, Finitely additive measures on the non-negative integers, Math. Scand. 24 (1969) 186-194. MR0274642 (43:405)

[4] R.A. Ryan, Dunford-Pettis Properties, Bull. Acad. Polonaise Sc. 27 (1979) 373-379. MR0557405 (80m:46018)

[5] J.D.M. Wright and K. Ylinen, Multilinear maps on products of operator algebras, J. Math. Anal. Appl. 292 (2004), 558-570 MR2047631

Department of Mathematics, University of Turku, Fin-20014 Turku, Finland

E-mail address: ylinen@utu.fi 